\title{
Constraints on the density perturbation spectrum from primordial black holes
}

\author{
Anne M. Green and Andrew R. Liddle \\ Astronomy Centre, University of Sussex, Falmer, Brighton BN1 9QH, U. K.
}

(February 5, 2008)

\begin{abstract}
We re-examine the constraints on the density perturbation spectrum, including its spectral index $n$, from the production of primordial black holes. The standard cosmology, where the Universe is radiation dominated from the end of inflation up until the recent past, was studied by Carr, Gilbert and Lidsey; we correct two errors in their derivation and find a significantly stronger constraint than they did, $n \lesssim 1.25$ rather than their 1.5. We then consider an alternative cosmology in which a second period of inflation, known as thermal inflation and designed to solve additional relic over-density problems, occurs at a lower energy scale than the main inflationary period. In that case, the constraint weakens to $n \lesssim 1.3$, and thermal inflation also leads to a 'missing mass' range, $10^{18} \mathrm{~g} \lesssim M \lesssim 10^{26} \mathrm{~g}$, in which primordial black holes cannot form. Finally, we discuss the effect of allowing for the expected non-gaussianity in the density perturbations predicted by Bullock and Primack, which can weaken the constraints further by up to 0.05 .
\end{abstract}

PACS numbers: $98.80 . \mathrm{Cq} \quad$ Sussex preprint SUSSEX-AST 97/4-2, astro-ph/9704251

\section{INTRODUCTION}

Primordial black holes (PBHs) are formed in the early universe if density perturbations are sufficiently large, and provide a useful probe of the primordial power spectrum over a wide range of scales. The data from COBE and from large-scale structure observations constrain the power spectrum on large scales (from about one megaparsec up to thousands of megaparsecs), whereas PBHs may form over a wide range of smaller scales (1 Mpc to $10^{-16} \mathrm{Mpc}$ ). Limits on their production can be used to constrain inflation models in which the perturbations grow as one moves to shorter scales, the so-called blue spectra.

There are a number of well-known limits, covering various mass ranges, on the maximum allowed mass fraction of PBHs [1 33]. Some are imposed at the present epoch and some at earlier stages such as nucleosynthesis. These constraints fall into two categories, those from the effects of Hawking radiation and those from the gravitational effects alone. The evaporation of PBHs via thermal emission has potentially observable astrophysical consequences, and while no unambiguous detection has been made, observations have placed limits on the maximum mass fraction of PBHs allowed at evaporation. PBHs with mass $M \lesssim 5 \times 10^{14} \mathrm{~g}$ will have evaporated before the present epoch. PBHs more massive than this will not have experienced significant evaporation, and their present density must not overclose the universe: $\Omega_{\mathrm{pbh}, 0}<1$. An additional, less secure, constraint on light PBHs can be obtained if one supposes that black hole evaporation leaves a stable relic, normally assumed to have a mass of order the Planck mass, rather than evaporating to nothing.

It is thought that PBHs can only form in the early universe, and that they do so at a time when the horizon mass equals the black hole mass. A number of formation mechanisms are possible [4,5], the simplest being formation from large density perturbations. The horizon mass in a radiation-dominated universe with temperature $T$ is given by

$$
M_{\mathrm{H}} \simeq 10^{18} \mathrm{~g}\left(\frac{10^{7} \mathrm{GeV}}{T}\right)^{2}
$$

from which we see that evaporating $\mathrm{PBHs}$ must form very early indeed in the history of the universe, in particular long before the epoch of nucleosynthesis $(T \sim 1 \mathrm{MeV})$ at which the standard big bang evolution is well validated.

In the 'standard' cosmology, the universe has been radiation dominated ever since the end of the reheating period after a phase of inflation at extremely high energies, which was responsible for the generation of density perturbations. Under this assumption, the limits on the PBH density can be extrapolated backwards to the time of formation to give limits on the initial mass fraction of PBHs, $\beta_{\mathrm{i}}=\rho_{\mathrm{pbh}, \mathrm{i}} / \rho_{\mathrm{tot}, \mathrm{i}}$ where $\rho_{\mathrm{pbh}, \mathrm{i}}$ and $\rho_{\mathrm{tot}, \mathrm{i}}$ are the $\mathrm{PBH}$ and total energy densities respectively, at the time at which the PBHs are formed. Substantial work has been done under this assumption [2, 3, 6]. However, there is no direct evidence requiring that the universe be radiation dominated at the high temperatures under consideration, and these limits can be greatly altered if the evolution of the Universe is more complex. In this paper we consider one of the most dramatic possible changes to the early evolution - the effect of a second period of inflation.

A recent extension to the standard cosmology is thermal inflation [7], a second period of inflation due to a scalar field known as a flaton. This has nothing to do with the normal period of inflation, which is still assumed to occur at some higher energy scale to solve the flatness and horizon problems and to generate density perturbations. Flaton fields, which are a consequence of supersymmetric theories, have a vacuum expectation value 
(vev) $M \gg 10^{3} \mathrm{GeV}$, even though their mass $m$ is only of order the supersymmetry scale, $\sim 10^{2}$ to $10^{3} \mathrm{GeV}$. Their potential is therefore almost flat. In the early universe these fields are held at zero by finite temperature effects, with false vacuum energy density $V_{0} \sim m^{2} M^{2}$. Once the temperature falls below $V_{0}^{1 / 4}$, the false vacuum energy density dominates the thermal energy density. This false vacuum energy begins to drive a new period of inflation. This inflation continues until the temperature drops to $T \sim m$, at which point thermal effects are no longer strong enough to anchor the flaton in the false vacuum. The most popular possible identity for flaton fields are the moduli fields in superstring theory.

A modulus field with a vev of order the Planck mass $m_{\mathrm{Pl}}$ (as is expected if the vev is non-zero) would produce many particles which would not decay before nucleosynthesis and hence would destroy the standard model of cosmology; this is the 'moduli problem' [8]. It cannot be solved in the same way as the monopole problem by invoking an early epoch of inflation lasting upwards of 60 $e$-foldings, since the energy scale at the end of inflation is normally greater than $10^{12} \mathrm{GeV}$ and the moduli would be regenerated after inflation. To avoid too many moduli being regenerated requires [9]

$$
V_{\text {inf }}^{1 / 4} \leq\left(10^{7} \text { to } 10^{8}\right) \mathrm{GeV}\left(\frac{1 \mathrm{GeV}}{T_{\mathrm{R}}}\right)^{1 / 4},
$$

where $T_{\mathrm{R}}$ is the reheat temperature. A single period of inflation at such a low energy scale would not be capable of producing the observed density perturbations, but a period of thermal inflation can solve this problem provided $M$ is within a range of several orders of magnitude around $10^{12} \mathrm{GeV}$. Taking $M \sim 10^{12} \mathrm{GeV}$ gives $V_{0}^{1 / 4} \sim 10^{7} \mathrm{GeV}$ so that around $\ln \left(10^{7} / 10^{3}\right) \sim 10 e$-foldings of thermal inflation occur, sufficient to dilute the moduli existing before thermal inflation but small enough to not affect the density perturbations generated during the first period of inflation.

The main effect of the period of thermal inflation is to dilute the density of $\mathrm{PBHs}$, relative to radiation, by a factor of $\left(a_{\mathrm{f}} / a_{\mathrm{i}}\right)^{3} \sim\left(10^{4}\right)^{3}$, where $a_{\mathrm{i}}$ and $a_{\mathrm{f}}$ are the scale factors immediately before and after thermal inflation. In addition, there are two more subtle changes from the standard scenario. Firstly, for $\mathrm{PBHs}$ which form in the time between the two periods of inflation, the comoving scale to which a given mass of $\mathrm{PBH}$ corresponds is changed. During thermal inflation the horizon mass remains constant whilst the comoving Hubble radius grows,

\footnotetext{
* Originally the flaton was taken to be the GUT Higgs 何 so that $M \sim M_{\mathrm{GUT}} \sim 10^{16} \mathrm{GeV}$ and $V_{0}^{1 / 4} \sim 10^{10} \mathrm{GeV}$, leading to $15 e$-foldings of inflation. However, successful nucleosynthesis requires $M \lesssim 10^{12} \mathrm{GeV}$ and thermalization of a stable lightest supersymmetric particle, if one exists, requires $M \lesssim 10^{10} \mathrm{GeV}$. 10 .
}

so a given $\mathrm{PBH}$ mass corresponds to a shorter comoving length scale and hence to a later stage in the original, density perturbation generating, epoch of inflation. Secondly, thermal inflation introduces a missing mass range of PBHs. This corresponds to those comoving scales which enter the horizon before thermal inflation (possibly forming black holes as they do so), and are then pulled back outside again during thermal inflation. Any new density perturbations might be expected to be small, since the energy scale of thermal inflation is much lower than the original inflationary period, and hence unable to form black holes when they re-enter the horizon again after thermal inflation. F From Eq. (11), this corresponds to masses in the range $10^{18} \mathrm{~g} \lesssim M \lesssim 10^{26} \mathrm{~g}$.

Finally, PBH formation requires $\delta \rho / \rho$ on the relevant scales to be two or three orders of magnitude larger than the value required by $\mathrm{COBE}$, which applies at very large scales. PBHs can therefore only be formed in significant numbers if the spectral index $n$ of the density perturbations is significantly above unity, normally referred to as a blue spectrum. Limits on PBH formation allow upper bounds to be placed on $n$; for the standard cosmology this was done by Carr et al. [3]. We shall first re-examine their calculation, and then generalize it to include the possibility of thermal inflation.

\section{PRIMORDIAL BLACK HOLE FORMATION}

In order for a $\mathrm{PBH}$ be formed, a collapsing overdense region must be large enough to overcome the pressure force resisting its collapse as it falls within its Schwarzschild radius. Consider a spherically symmetric region with density $\tilde{\rho}$ greater than that of the background, whose evolution will be governed by the positive curvature Friedmann equation [11]. The perturbed region stops expanding when $\tilde{H}=0$, at which time the region has size $R_{\mathrm{c}} \approx \delta_{\mathrm{i}}^{-1 / 2} R_{\mathrm{i}}$ where $R_{\mathrm{i}}$ is its size at some arbitrary initial time and $\delta_{\mathrm{i}}=\left(\tilde{\rho}_{\mathrm{i}}-\rho_{\mathrm{i}}\right) / \rho_{\mathrm{i}}$ is the initial density perturbation [12]. If the perturbed region contains enough matter to overcome any pressure forces, it will continue to contract. This requires that its radius exceeds the Jeans length, $R_{\mathrm{c}} \geq R_{\text {Jeans }}=c_{\mathrm{s}} t_{\mathrm{c}}$, where $c_{\mathrm{s}}$ is the sound speed. In a radiation-dominated universe, $R_{\text {Jeans }} \sim t_{\mathrm{c}} / \sqrt{3}$. Now $R_{\mathrm{c}} / t_{\mathrm{c}} \approx R_{\mathrm{i}} / t_{\mathrm{i}} \delta_{\mathrm{i}}^{-1 / 2}$, and since this expression is constant with time we can evaluate it at horizon crossing $(R=t)$ leading to a constraint on the perturbations at horizon crossing $\delta \geq 1 / 3$. There is also an upper limit of $\delta \leq 1$; a perturbation which exceeded this value would correspond initially to a separate closed

\footnotetext{
${ }^{\dagger}$ Note however the standard perturbation calculation breaks down as the hypothesis that the initial state is the vacuum probably cannot be justified. It is not clear how to make the necessary generalization.
} 
universe [13:5], which is an inconsistent initial condition for our purposes. So for PBH formation we require the initial fluctuations to satisfy

$$
1 / 3 \leq \delta \leq 1 .
$$

When a perturbation satisfying the above condition crosses the horizon, a PBH will be formed with mass [5]

$$
M=\gamma^{3 / 2} M_{\mathrm{H}}=\frac{\gamma^{3 / 2}}{g_{\star \text { form }}^{1 / 2}}\left(\frac{t}{t_{\mathrm{Pl}}}\right) m_{\mathrm{Pl}},
$$

where the background equation of state is $p=\gamma \rho, \gamma$ being $1 / 3$ in a radiation-dominated universe. Here

$$
M_{\mathrm{H}}=\frac{4 \pi}{3} \rho\left(H^{-1}\right)^{3},
$$

and $g_{\star}$ is the effective number of massless degrees of freedom at this time. We define $\beta_{\mathrm{i}}$ to be the initial mass fraction of $\mathrm{PBHs}$, which is given by the fraction of the Universe satisfying Eq. (3):

$$
\beta_{i} \equiv \frac{\rho_{\mathrm{pbh}, \mathrm{i}}}{\rho_{\mathrm{tot}, \mathrm{i}}}=\int_{1 / 3}^{1} p(\delta) \mathrm{d} \delta,
$$

where $p(\delta)$ is the probability distribution for $\delta$.

Normally (for instance in large-scale structure studies) when one considers perturbations the probability distribution is assumed to be gaussian. This is well justified when the perturbations are small. However, Bullock and Primack 11] have recently challenged this assumption for PBH formation, since the perturbations cannot be very small if a significant formation rate is to be obtained. They typically find a suppression of large perturbations relative to the gaussian hypothesis. While this suppression can be very dramatic when expressed in terms of the number of black holes formed, it actually does not lead to a large change in constraints on the perturbation spectrum. We shall therefore maintain the gaussian assumption for our derivations, and in Section $\mathrm{V}$ we shall assess the changes non-gaussianity introduces.

In order to examine specific mass ranges, we have to smooth the density distribution, which is done in the normal way using a window function $W(k R)$, which we take to be a top-hat. For gaussian distributed fluctuations, the probability distribution of the smoothed density field, $p(\delta(M))$, is given by

$$
p(\delta(M)) \mathrm{d} \delta(M)=\frac{1}{\sqrt{2 \pi} \sigma(M)} \exp \left(-\frac{\delta^{2}(M)}{2 \sigma^{2}(M)}\right) \mathrm{d} \delta(M) .
$$

Here $\sigma(M)$ is the mass variance evaluated at horizon crossing, defined for example in Ref. [14]

$$
\sigma^{2}(M)=\frac{1}{2 \pi^{2}} \int_{0}^{\infty} P(k) W^{2}(k R) k^{2} \mathrm{~d} k .
$$

where $P(k)=\left\langle\left|\delta_{k}\right|^{2}\right\rangle$ is the power spectrum, the $\delta_{k}$ being the coefficients when $\delta(x)$ is Fourier expanded. The power spectrum is usually taken to have primordial form $P(k) \propto k^{n}$ for simplicity; in general there is no reason to expect inflation models to produce power-law spectra over as wide as range of scales as we will need to consider, though there are models which do.

The mass fraction of black holes is given from the above by

$$
\beta(M)=\int_{1 / 3}^{1} \frac{1}{\sqrt{2 \pi} \sigma(M)} \exp \left(-\frac{\delta^{2}}{2 \sigma^{2}(M)}\right) \mathrm{d} \delta .
$$

Since the integrand is a rapidly falling function in the regime of interest, dropping by a factor $\exp (-0.5)$ every time $\delta$ is increased by $\sigma$, this integral can be approximated by evaluating the integrand at $\delta=1 / 3$ and multiplying by $\sigma(M)$, leading to

$$
\beta(M) \approx \sigma(M) \exp \left(-\frac{1}{18 \sigma^{2}(M)}\right) .
$$

Strictly speaking, this is the mass fraction in black holes of mass greater than $M$, but in practice $\beta(M)$ is such a rapidly falling function that these can be taken to all have the same mass $M$.

The final step is to relate the mass scales to comoving scales during inflation. In the notation of Ref. [14], the initial spectrum of perturbations is $\delta_{\mathrm{H}}^{2}(k) \propto k^{n-1}$ where

$$
\delta_{\mathrm{H}}^{2}=\left(\frac{k^{3}}{2 \pi^{2}}\right)\left(\frac{a H}{k}\right)^{4} P(k) ;
$$

The quantity $\delta_{\mathrm{H}}$ stays constant on scales above the Hubble radius and is a good estimate of the rms density contrast at horizon entry [14]. During radiation domination the comoving Hubble radius $H^{-1} / a$ is proportional to $T^{-1}$, so that a given scale $k$ crosses within the Hubble radius when

$$
k^{-1}=\frac{H^{-1}}{a}=\left(\frac{H^{-1}}{a}\right)_{\mathrm{eq}}\left(\frac{T_{\mathrm{eq}}}{T}\right),
$$

where subscript 'eq' refers to quantities evaluated at matter-radiation equality. Meanwhile the horizon mass $M_{\mathrm{H}}$, Eq. (5), varies as $T^{-2}$ so that, in the absence of thermal inflation, we get

$$
k^{-1}=\left(\frac{H^{-1}}{a}\right)_{\text {eq }}\left(\frac{M_{\mathrm{H}}}{M_{\mathrm{H}, \mathrm{eq}}}\right)^{1 / 2} .
$$

Substituting this into the expression for $\delta_{\mathrm{H}}(k)$ we obtain

$$
\sigma_{\text {hor }}(M)=\sigma_{\text {hor }}\left(M_{\mathrm{eq}}\right)\left(\frac{M}{M_{\mathrm{eq}}}\right)^{(1-n) / 4},
$$

where $M_{\text {eq }}$ is the horizon mass at matter-radiation equality. We stress that this equation refers to the dispersion 


\begin{tabular}{|c|c|c|}
\hline \hline Constraint & Range & Reason \\
\hline \hline$\alpha_{\text {evap }}<0.04$ & $10^{9} \mathrm{~g}<M<10^{13} \mathrm{~g}$ & Entropy per baryon at nucleosynthesis \\
\hline$\alpha_{\text {evap }}<10^{-26}\left(M / m_{\mathrm{Pl}}\right)$ & $M=5 \times 10^{14} \mathrm{~g}$ & $\gamma$ rays from current explosions \\
\hline$\alpha_{\text {evap }}<6 \times 10^{-10}\left(M / m_{\mathrm{Pl}}\right)^{1 / 2}$ & $10^{9} \mathrm{~g}<M<10^{11} \mathrm{~g}$ & n̄ production at nucleosynthesis \\
\hline$\alpha_{\text {evap }}<5 \times 10^{-29}\left(M / m_{\mathrm{Pl}}\right)^{3 / 2}$ & $10^{10} \mathrm{~g}<M<10^{11} \mathrm{~g}$ & Deuterium destruction \\
\hline$\alpha_{\text {evap }}<1 \times 10^{-59}\left(M / m_{\mathrm{Pl}}\right)^{7 / 2}$ & $10^{11} \mathrm{~g}<M<10^{13} \mathrm{~g}$ & Helium-4 spallation \\
\hline \hline
\end{tabular}

TABLE I. Limits on the mass fraction of PBHs at evaporation.

at horizon crossing, not at constant time.

The lightest black holes to form are those which enter the horizon immediately after inflation. For simplicity we shall assume prompt reheating, and Eq. (1) then gives the minimum mass. Carr et al. [3] examine some consequences of delayed reheating.

\section{LIMITS ON THE PBH ABUNDANCE}

\section{A. At evaporation}

The observational constraints on the mass fraction of black holes at evaporation, $\alpha_{\text {evap }}(M)=\rho_{\text {pbh }} / \rho_{\text {rad }}$ are well known [1]3], and are listed in Table [.

To interpret these we need to relate the black hole mass to their lifetime. Carr [5] parametrizes the results of Page [15], which were found numerically by considering the number of species which a black hole of given mass can emit at a significant rate, to give the following relation between PBH mass and lifetime

$$
\tau_{\text {evap }}=\frac{9 \times 10^{-27}}{f(M)}\left(\frac{M}{1 \mathrm{~g}}\right)^{3} \mathrm{sec},
$$

where $f(M)$ depends on the number of particle species which can be emitted and is normalized to 1 for holes which emit only massless particles. Note that the bulk of the evaporation always takes place near the initial temperature, so in this expression one only needs $f$ at the initial mass and not as a time-varying quantity. Considering the number of spin states available for a PBH to evaporate into at the present day $\left(g_{\text {eff }, 0}\right)$; there are two polarizations of photon plus three neutrino species which each have two spin states and give a contribution $7 / 8$ times

$\ddagger$ This disagrees with Ref. [3], in which a different scaling $\sigma(M) \propto M^{(1-n) / 6}$ was used. This arises from assuming $M \propto k^{-3}$ with no time-dependence - i.e. that the comoving mass density is conserved. This is true for matter domination but not for radiation domination where the comoving mass density decreases with time. Because our scaling is stronger with $n$, our final constraints on $n$ are tighter than theirs. that of the photons, since they obey Fermi rather than Bose statistics. Therefore $g_{\text {eff }, 0}=2+(3 \times 2 \times 7 / 8)=7.25$ so that $f(M) \equiv g_{\text {eff }, 0} / 7.25$ and

$$
\tau_{\text {evap }}=\frac{1.2 \times 10^{4}}{g_{\mathrm{eff}}}\left(\frac{M}{m_{\mathrm{Pl}}}\right)^{3} t_{\mathrm{Pl}},
$$

with the value of $g_{\text {eff }}$ at the time of evaporation being taken. For $M>4 \times 10^{9} \mathrm{~g}$ the temperature at evaporation is sufficiently low $\left(<10^{-4} \mathrm{GeV}\right)$ that $g_{\text {eff }}$ has its present day value of 7.25. Eq. (16) is often quoted without the factor of $1.2 \times 10^{4}$; this is certainly non-negligible when calculating $\mathrm{PBH}$ lifetime although other approximations often made when limiting the initial $\mathrm{PBH}$ abundance (e.g. $g_{\text {eff }} \sim 1, \gamma \sim 1$ and $M \sim M_{\mathrm{H}}$ in Ref. [3]) appear to largely cancel this factor.

\section{B. Present-day PBH density}

From Eq. (16), PBHs of mass $M>5 \times 10^{14} \mathrm{~g}$ will not have evaporated by the present day, but their initial abundance can be constrained from the fact that their present mass density must not overclose the universe

$$
\Omega_{\mathrm{pbh}, 0}=\Omega_{\mathrm{pbh}, \mathrm{eq}}<1 .
$$

\section{Present-day relic density}

It has been argued [16] that PBHs may not evaporate completely, as originally assumed, but instead leave a relic with mass $M_{\text {rel }} \sim m_{\mathrm{Pl}}$. If this is the case the present mass density of relics, which will remain from all PBHs with initial mass $M<5 \times 10^{14} \mathrm{~g}$, similarly must not overclose the universe, leading to

$$
\begin{aligned}
\Omega_{\mathrm{rel}, 0} & =\Omega_{\mathrm{rel}, \mathrm{eq}}=\left(\frac{m_{\mathrm{Pl}}}{M}\right) \frac{\rho_{\mathrm{pbh}, \mathrm{i}}}{\rho_{\mathrm{tot}, \mathrm{eq}}}\left(\frac{T_{0}}{T_{\mathrm{form}}}\right)^{3} \\
& =\left(\frac{m_{\mathrm{Pl}}}{M}\right) \frac{\beta_{\mathrm{i}}}{1-\beta_{\mathrm{i}}} \frac{\rho_{\mathrm{rad}, \mathrm{i}}}{\rho_{\mathrm{tot}, \mathrm{eq}}}\left(\frac{T_{0}}{T_{\mathrm{form}}}\right)^{3}<1 .
\end{aligned}
$$




\begin{tabular}{|c|c|}
\hline \hline Constraint & Range \\
\hline$\alpha_{\mathrm{i}}<3 \times 10^{-16}\left(10^{9} \mathrm{~g} / M\right)$ & $10^{9} \mathrm{~g}<M<10^{13} \mathrm{~g}$ \\
\hline$\alpha_{\mathrm{i}}<3 \times 10^{-27}$ & $M \simeq 5 \times 10^{14} \mathrm{~g}$ \\
\hline$\alpha_{\mathrm{i}}<3 \times 10^{-17}\left(10^{9} \mathrm{~g} / M\right)^{1 / 2}$ & $10^{9} \mathrm{~g}<M<10^{11} \mathrm{~g}$ \\
\hline$\alpha_{\mathrm{i}}<3 \times 10^{-22}\left(M / 10^{10} \mathrm{~g}\right)^{1 / 2}$ & $10^{10} \mathrm{~g}<M<10^{11} \mathrm{~g}$ \\
\hline$\alpha_{\mathrm{i}}<3 \times 10^{-21}\left(M / 10^{9} \mathrm{~g}\right)^{5 / 2}$ & $10^{11} \mathrm{~g}<M<10^{13} \mathrm{~g}$ \\
\hline$\alpha_{\mathrm{i}}<1 \times 10^{-19}\left(M / 10^{15} \mathrm{~g}\right)^{1 / 2}$ & $M>10^{15} \mathrm{~g}$ \\
\hline$\alpha_{\mathrm{i}}<0.1\left(M / 10^{15} \mathrm{~g}\right)^{3 / 2}$ & $M<10^{15} \mathrm{~g}$ \\
\hline \hline
\end{tabular}

TABLE II. Limits on the initial mass fraction of PBHs, without thermal inflation. We define $\alpha_{\mathrm{i}} \equiv \beta_{\mathrm{i}} /\left(1-\beta_{\mathrm{i}}\right)$ for compactness. The final constraint is the relic constraint which only applies if relics are assumed.

\section{LIMITS ON INITIAL MASS FRACTION OF PBHS}

\section{A. Standard evolution of the universe}

In constraining the initial mass function of the black holes, one needs to assume an entire history for the universe from the time of formation to the present. Traditionally, it has been assumed that the universe was radiation dominated up until the recent matter-dominated era, and then the limits of Section [II] can easily be evolved backwards in time in order to constrain the initial mass fraction of PBHs. The energy density in radiation dilutes as $\rho_{\text {rad }} \propto a^{-4}$, whereas that in PBHs decreases more slowly, $\rho_{\mathrm{pbh}} \propto a^{-3}$. Therefore

$$
\left(\frac{\rho_{\mathrm{pbh}}}{\rho_{\mathrm{rad}}}\right)_{\text {evap }}=\alpha(M)_{\text {evap }}=\frac{\beta_{\mathrm{i}}}{1-\beta_{\mathrm{i}}}\left(\frac{t_{\text {evap }}}{t_{\text {form }}}\right)^{1 / 2} .
$$

Using Eqs. (14) and (16), and taking $g_{\star} \sim 100$

$$
\alpha(M)_{\mathrm{evap}}=3.2 \frac{\beta_{\mathrm{i}}}{1-\beta_{\mathrm{i}}}\left(\frac{M}{m_{\mathrm{Pl}}}\right) .
$$

The gravitational constraint can be evaluated simply:

$$
\begin{aligned}
\rho_{\mathrm{PBH}, \mathrm{eq}} & =\rho_{\mathrm{pbh}, \mathrm{i}}\left(\frac{T_{\mathrm{eq}}}{T_{\text {form }}}\right)^{3} \\
& =\frac{\beta_{\mathrm{i}}}{1-\beta_{\mathrm{i}}} \frac{\pi^{2}}{30} g_{\star}^{\text {form }} T_{\text {form }}^{4}\left(\frac{T_{\mathrm{eq}}}{T_{\text {form }}}\right)^{3},
\end{aligned}
$$

and

$$
\rho_{\text {tot }, \mathrm{eq}}=2 \frac{\pi^{2}}{30} g_{\star}^{\mathrm{eq}} T_{\mathrm{eq}}^{4},
$$

where $g_{\star}^{\text {form }} \sim 100$ and $g_{\star}^{\text {eq }} \sim 3$, , s so that

\footnotetext{
${ }^{\S}$ Note that $g_{\star}$ is the effective number of degrees of freedom
}

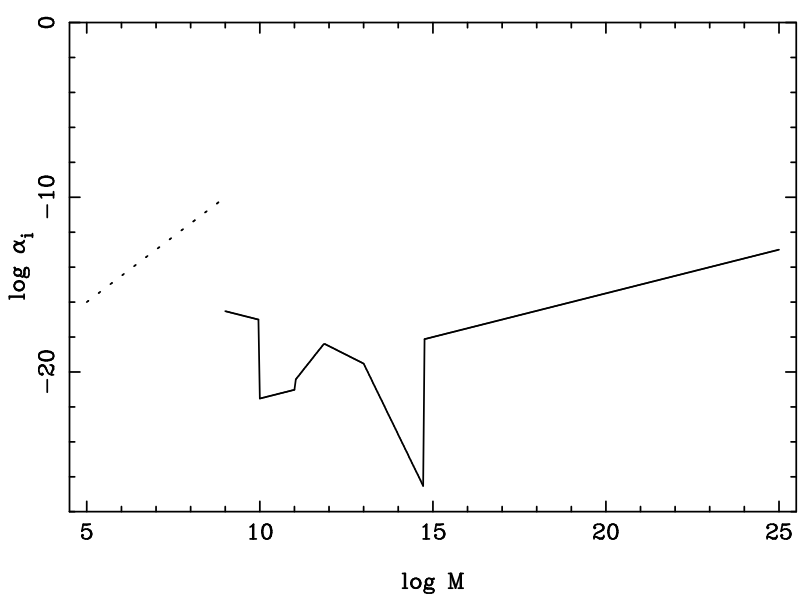

FIG. 1. The tightest limits on the initial mass fraction of PBHs, $\alpha_{\mathrm{i}}$. The relic constraint is shown as a dotted line, emphasizing that it is not compulsory.

$$
\Omega_{\mathrm{pbh}, 0}=17 \frac{\beta_{\mathrm{i}}}{1-\beta_{\mathrm{i}}}\left(\frac{t_{\text {form }}}{t_{\mathrm{eq}}}\right)^{1 / 2} .
$$

Taking $t_{\text {eq }}=t_{0} \Omega_{\text {rel }}^{3 / 2}=6.5 h^{-1} \mathrm{Gyr} \times\left(4 \times 10^{-5} \mathrm{~h}^{-2}\right)^{3 / 2}$ and using Eq. (四)

$$
\Omega_{\mathrm{pbh}, 0}=6.7 \times 10^{28} \frac{\beta_{\mathrm{i}}}{1-\beta_{\mathrm{i}}}\left(\frac{m_{\mathrm{Pl}}}{M}\right)^{1 / 2} .
$$

so that the constraint $\Omega_{\mathrm{pbh}, 0}<1$ leads to

$$
\frac{\beta_{\mathrm{i}}}{1-\beta_{\mathrm{i}}}<1.5 \times 10^{-29}\left(\frac{M}{m_{\mathrm{Pl}}}\right)^{1 / 2} .
$$

The calculation for the relic limit can be carried out identically leading to

$$
\frac{\beta_{\mathrm{i}}}{1-\beta_{\mathrm{i}}}<1.5 \times 10^{-29}\left(\frac{M}{m_{\mathrm{Pl}}}\right)^{3 / 2} .
$$

with the extra factor of $\left(M / m_{\mathrm{Pl}}\right)$ from Eq. (18).

The various limits on the initial mass fraction of $\mathrm{PBHs}$ are displayed in Table III and illustrated in Fig. 1.

\section{B. With a period of thermal inflation}

We model the period of thermal inflation by assuming that at $T=10^{7} \mathrm{GeV}$ the energy density in radiation splits into two components, with one degree of freedom becoming the inflaton and the remainder staying as radiation:

as far as cosmology is concerned, evaluated at the photon temperature. It is lower than $g_{\text {eff }}$ quoted earlier since the cosmic neutrino background is at a lower temperature than the microwave background. 


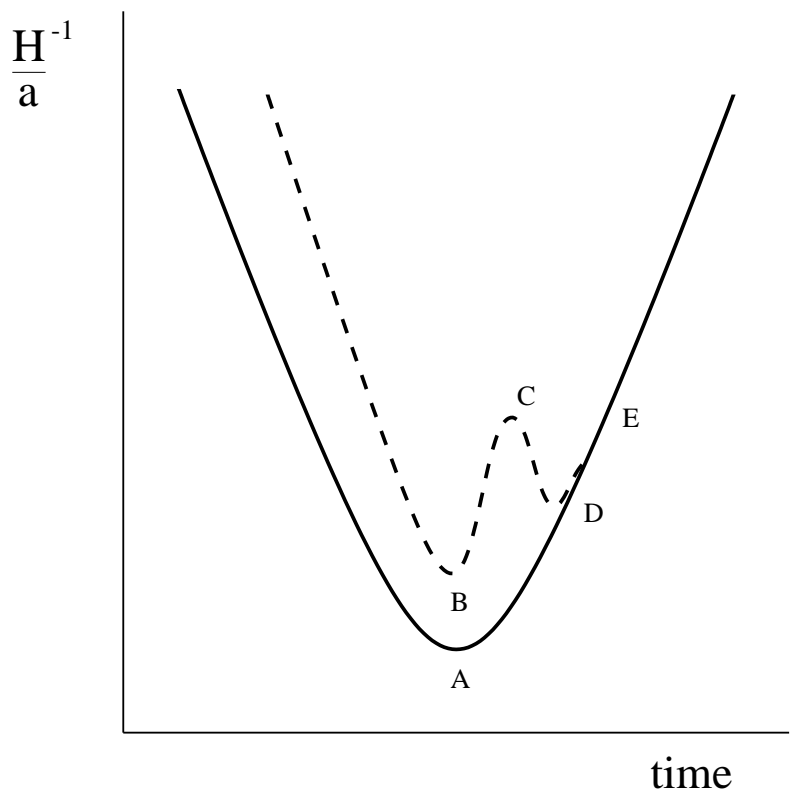

FIG. 2. A schematic of the variation of the comoving Hubble radius $\left(H^{-1} / a\right)$ with time for the standard evolution of the universe (solid line) and with thermal inflation (dashed line). Points $\mathrm{A}$ and $\mathrm{B}$ correspond to the end of the original period of inflation in the standard evolution and with thermal inflation respectively. Thermal inflation begins at point $\mathrm{C}$ and finishes at D, after which time the comoving Hubble radii must coincide. Between $\mathrm{D}$ and $\mathrm{E}$ the scales which are entering the Hubble radius are doing so for the second time so that no PBHs are formed in this region. The values of the comoving Hubble radius and the horizon mass at these points are displayed in Table III. We denote the current comoving Hubble radius and horizon mass as $\left(H^{-1} / a\right)_{0}$ and $M_{\mathrm{Ho}}$ respectively.

$$
\rho_{\mathrm{rad}} \rightarrow \tilde{\rho}_{\mathrm{rad}}+\rho_{\phi},
$$

where $\rho_{\phi}=\pi^{2}\left(10^{7} \mathrm{GeV}\right)^{4} / 30$ is the false energy density of the flaton field which drives thermal inflation, i.e.

$$
\frac{\pi^{2}}{30} g_{\star} T^{4} \rightarrow \frac{\pi^{2}}{30}\left(g_{\star}-1\right) T^{4}+\rho_{\phi} .
$$

Thermal inflation proper then commences once the radiation has redshifted sufficiently, at

$$
T_{\mathrm{ti}}=\frac{10^{7} \mathrm{GeV}}{\left(g_{\star}-1\right)^{1 / 4}},
$$

when $\rho_{\phi}>\tilde{\rho}_{\text {rad }}$ and continues until the flaton field rolls to its true vacuum state at $T=10^{3} \mathrm{GeV}$. We then assume that reheating is efficient so that the universe is reheated to $T_{\mathrm{ti}}$ and the subsequent evolution has its standard form. Inefficient reheating, normally modelled as matter domination, would make little difference. The duration of thermal inflation is negligible in terms of the evaporation time-scale, so its dominant effect on $\mathrm{PBH}$ evolution is to dilute their energy density relative to that of radiation. The radiation energy density is the same before

\begin{tabular}{|c|c|c|}
\hline \hline Point & Comoving Hubble Radius & Horizon Mass \\
\hline A & $\left(\frac{H^{-1}}{a}\right)_{0} \frac{T_{0}}{T_{\mathrm{RH}}}$ & $M_{\mathrm{H}, 0}\left(\frac{T_{0}}{T_{\mathrm{RH}}}\right)^{2}$ \\
\hline B & $10^{4}\left(\frac{H^{-1}}{a}\right)_{0} \frac{T_{0}}{T_{\mathrm{RH}}}$ & $M_{\mathrm{H}, 0}\left(\frac{T_{0}}{T_{\mathrm{RH}}}\right)^{2}$ \\
\hline C & $\left(\frac{H^{-1}}{a}\right)_{0} \frac{T_{0}}{10^{3} \mathrm{GeV}}$ & $M_{\mathrm{H}, 0}\left(\frac{T_{0}}{10^{7} \mathrm{GeV}}\right)^{2}$ \\
\hline D & $\left(\frac{H^{-1}}{a}\right)_{0} \frac{T_{0}}{10^{7} \mathrm{GeV}}$ & $M_{\mathrm{H}, 0}\left(\frac{T_{0}}{10^{7} \mathrm{GeV}}\right)^{2}$ \\
\hline E & $\left(\frac{H^{-1}}{a}\right)_{0} \frac{T_{0}}{10^{3} \mathrm{GeV}}$ & $M_{\mathrm{H}, 0}\left(\frac{T_{0}}{10^{3} \mathrm{GeV}}\right)^{2}$ \\
\hline \hline
\end{tabular}

TABLE III. Comoving Hubble Radii and horizon masses at points on Fig. 8 .

and after thermal inflation (assuming efficient reheating) whilst the energy density of PBHs is diluted by a factor $\rho_{\mathrm{i}} / \rho_{\mathrm{f}}=\left(a_{\mathrm{f}} / a_{\mathrm{i}}\right)^{3} \sim\left(10^{4}\right)^{3}$, where $a_{\mathrm{i}}$ and $a_{\mathrm{f}}$ are the scale factors immediately before and after thermal inflation.

Fig. 目illustrates the variation of the comoving Hubble radius both with and without thermal inflation, with the Hubble radius and $M_{\mathrm{H}}$ at important points being given in Table III.

Provided that the PBHs do not come to dominate before thermal inflation, the limits on the initial black hole mass fraction from the evaporation constraints are simply weakened by a factor of $10^{12}$. The condition for the universe to be radiation dominated before thermal inflation commences is

$$
\left(\frac{\beta}{1-\beta}\right)_{\mathrm{T}_{\mathrm{ti}}}=\frac{\beta_{\mathrm{i}}}{1-\beta_{\mathrm{i}}}\left(\frac{T_{\mathrm{form}}}{T_{\mathrm{ti}}}\right)<1 .
$$

Using Eq. (画) this requires

$$
\frac{\beta_{\mathrm{i}}}{1-\beta_{\mathrm{i}}}<6 \times 10^{-12}\left(\frac{M}{m_{\mathrm{Pl}}}\right)^{1 / 2} .
$$

If the PBHs come to dominate at $T>10^{7} \mathrm{GeV}$ then thermal inflation can only commence once the false energy density of the flaton field dominates the energy density on PBHs, $\rho_{\phi}>\rho_{\mathrm{pbh}}$. This delays the start of inflation, so that a smaller number of $e$-foldings of inflation occur; however, the dilution of the $\mathrm{PBH}$ energy density relative to that of radiation remains the same. During the intermediate period $\rho_{\phi}$ remains constant while $\rho_{\mathrm{pbh}} \propto a^{-3}$, so $\rho_{\mathrm{pbh}}$ is rapidly reduced to below $\rho_{\phi}$ and the resulting constraints from Hawking radiation on the initial mass fraction are only slightly tighter than if thermal inflation commences at $T_{\mathrm{ti}}=10^{7} / 3 \mathrm{GeV}$. However these limits are tighter than Eq. (31), so that in fact PBHs with $M>10^{9} \mathrm{~g}$ cannot be produced with sufficient abundance that they come to dominate the universe before thermal inflation.

In the case of lighter PBHs, which are only constrained by the present-day relic density, the condition for radiation domination before thermal inflation commences, 


\begin{tabular}{|c|c|}
\hline \hline Constraint & Range \\
\hline$\alpha_{\mathrm{i}}<3 \times 10^{-4}\left(10^{9} \mathrm{~g} / M\right)$ & $10^{9} \mathrm{~g}<M<10^{13} \mathrm{~g}$ \\
\hline$\alpha_{\mathrm{i}}<3 \times 10^{-15}$ & $2 \times 10^{14} \mathrm{~g}<M<5 \times 10^{14} \mathrm{~g}$ \\
\hline$\alpha_{\mathrm{i}}<3 \times 10^{-5}\left(10^{9} \mathrm{~g} / M\right)^{1 / 2}$ & $10^{9} \mathrm{~g}<M<10^{11} \mathrm{~g}$ \\
\hline$\alpha_{\mathrm{i}}<3 \times 10^{-10}\left(M / 10^{10} \mathrm{~g}\right)^{1 / 2}$ & $10^{10} \mathrm{~g}<M<10^{11} \mathrm{~g}$ \\
\hline$\alpha_{\mathrm{i}}<3 \times 10^{-9}\left(M / 10^{9} \mathrm{~g}\right)^{5 / 2}$ & $10^{11} \mathrm{~g}<M<10^{13} \mathrm{~g}$ \\
\hline$\alpha_{\mathrm{i}}<1 \times 10^{11}\left(M / 10^{15} \mathrm{~g}\right)^{3 / 2}$ & $M<10^{15} \mathrm{~g}$ \\
\hline$\alpha_{\mathrm{i}}<1 \times 10^{-7}\left(M / 10^{15} \mathrm{~g}\right)^{1 / 2}$ & $10^{15} \mathrm{~g}<M<10^{18} \mathrm{~g}$ \\
\hline$\alpha_{\mathrm{i}}<1 \times 10^{-19}\left(M / 10^{15} \mathrm{~g}\right)^{1 / 2}$ & $M>10^{26} \mathrm{~g}$ \\
\hline \hline
\end{tabular}

TABLE IV. Limits on initial mass fraction of $\mathrm{PBHs}$ if thermal inflation occurs

Eq. (31), is more constraining than the requirement that $\Omega_{\text {rel, }, 0}<1$. These light PBHs can therefore come to dominate before thermal inflation and delay its start, as discussed above, although the resulting constraint from $\Omega_{\mathrm{rel}, 0}<1$ is virtually the same as when the universe is radiation dominated at the start of thermal inflation.

PBHs with mass $M<10^{18} \mathrm{~g}$ are formed before thermal inflation and their energy densities will therefore be diluted by thermal inflation, so that the gravitational constraint for $10^{15} \mathrm{~g}<M<10^{18} \mathrm{~g}$ and the relic constraint will be weakened by a factor $10^{12}$. This allows PBHs with $M<10^{9} \mathrm{~g}$ to be produced with initial abundance $\beta_{\mathrm{i}}$ close to one, although not arbitrarily so since sufficient thermal inflation to dilute the present day relic density must occur. During inflation $M_{\mathrm{H}}$ remains constant before increasing as $T^{-2}$ again after inflation; however, until the temperature falls to $T=10^{3} \mathrm{GeV}$ once more the scales that are entering the Hubble radius will be doing so for the second time having first entered at $T>T_{\mathrm{ti}}$ (and possibly forming black holes) before being inflated away again. There are therefore no new density perturbations present to collapse into $\mathrm{PBHs}$, so whilst $T$ falls from $10^{7} \mathrm{GeV}$ to $10^{3} \mathrm{GeV}$ after thermal inflation no PBHs form leading to a 'missing' mass range $10^{18} \mathrm{~g}<M<10^{26} \mathrm{~g}$. The gravitational constraints on PBHs with mass $M>10^{26} \mathrm{~g}$, which form after thermal inflation, are unchanged.

The various limits on the initial mass fraction of PBHs are displayed in Table $\mathrm{IV}$ and illustrated in Fig. 3 .

\section{LIMITS ON THE SPECTRAL INDEX}

Using Eq. (14), the limits on the initial mass fraction can be used to constrain the spectral index of the density perturbations $n$. The four-year fitting function to the COBE data, assuming negligible contribution from gravitational waves, gives the normalization at the present Hubble scale $\left(k=a_{0} H_{0}\right)$ [17

$$
\delta_{\mathrm{H}}(\mathrm{n})=1.91 \times 10^{-5} \exp [1.01(1-\mathrm{n})] .
$$

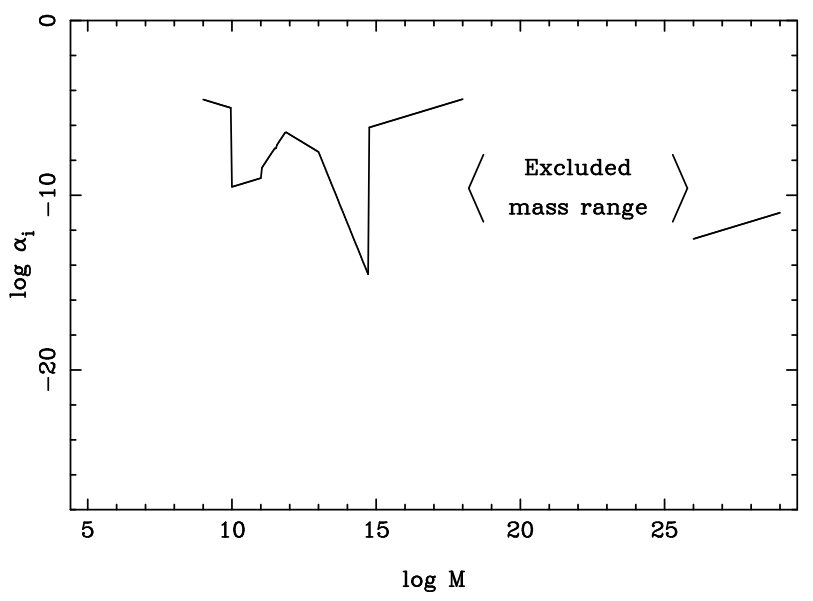

FIG. 3. The tightest limits on the initial mass fraction of PBHs $\alpha_{\mathrm{i}}$ if thermal inflation occurs, on the same vertical scale as Fig. 11. The gap $10^{18} \mathrm{~g}<M<10^{26} \mathrm{~g}$ is the excluded mass range. For $M<10^{9} \mathrm{~g}$ a large initial mass fraction $\sim 1$ of PBHs is allowed.

When inserted in Eq. (8) and numerically integrated, this allows us to normalize $\sigma(M)$ for a chosen fixed $M$. We take the COBE data to correspond to a scale equal to the present Hubble radius $R_{0}=3000 h^{-1}$, so that $M_{0}=1 \times 10^{56} \mathrm{~g}$ is the present horizon mass which gives a normalization $\sigma\left(10^{56} \mathrm{~g}\right)=9.5 \times 10^{-5}$. To the level of accuracy at which we are working $\sigma\left(10^{56} \mathrm{~g}\right)$ varies only slowly with $n$. Putting this in Eq. (14) gives

$$
\sigma_{\text {hor }}(M)=9.5 \times 10^{-5}\left(\frac{M}{10^{56} \mathrm{~g}}\right)^{(1-n) / 4},
$$

Here we ignore the change in slope to $(1-n) / 6$ which occurs at matter domination (corresponding to a mass $M_{0} t_{\text {eq }} / t_{0} \simeq 10^{49} \mathrm{~g}$ ), which only changes the constraint on $n$ by about 0.01 .

Taking logarithms in Eq. (10) gives

$$
\sigma(M)=0.15\left(\log _{10} \sigma(M)-\log _{10} \beta_{i}\right)^{-1 / 2} .
$$

Since $\beta_{\mathrm{i}} /\left(1-\beta_{\mathrm{i}}\right) \ll 1$, we can take $\beta_{\mathrm{i}} \approx \beta_{\mathrm{i}} /\left(1-\beta_{\mathrm{i}}\right)$ and find the upper bounds on $n$ corresponding to each of the limits found in the previous section.

\section{A. Standard evolution of the Universe}

The appropriate constraint is normally determined by the lightest PBHs that can form, given by Eq. (11). The tightest limit is $n<1.22$ from the deuterium destruction constraint evaluated at $M \sim 10^{11} \mathrm{~g}$, although all the constraints due to the evaporation of the PBHs require $n<1.24$. The tightest constraint from the limit on the present density of PBHs is $n<1.31$ at $M \sim 10^{15} \mathrm{~g}$, weakening with increasing $M$. The relic constraint may place a stronger constraint on $n$, depending on the minimum mass of PBHs produced, which is determined by 


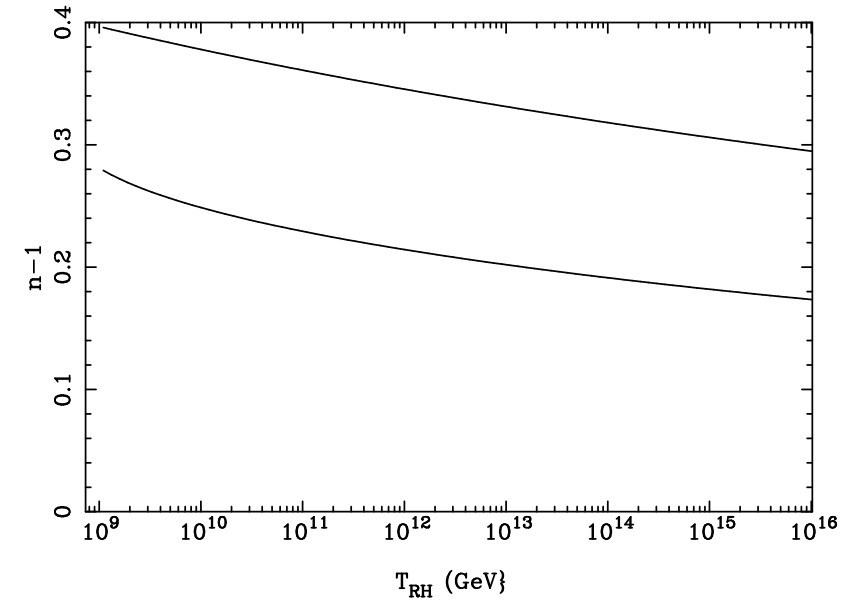

FIG. 4. The variation of the limits on $n$ with reheating temperature from the relic constraint (lower line) and from $\delta\left(M_{\min }\right)<1$.

the reheating temperature $T_{\mathrm{RH}}$ after the initial period of conventional inflation

$$
M_{\min }=M_{0}\left(\frac{T_{0}}{T_{\mathrm{RH}}}\right)^{2} .
$$

If $T_{\mathrm{RH}}<10^{9} \mathrm{GeV}$, there is not sufficient time for $\rho_{\mathrm{PBH}}$ (and after evaporation $\rho_{\text {rel }}$ ) to increase by enough relative to $\rho_{\text {rad }}$ for the relics to dominate today. For comparison, in Fig. A we plot the variation of the limit on $n$ with reheating temperature and the limit from the simple requirement that $\delta\left(M_{\min }\right)<1$, where $M_{\min }=$ $\left(T_{\mathrm{Pl}} / T_{\mathrm{RH}}\right)^{2} m_{\mathrm{Pl}}$ is the mass of the lightest PBHs formed immediately after the first period of inflation.

In summary, the tightest Hawking radiation constraint on $n$ we obtain is $n<1.22$, although if the reheating temperature is sufficiently high the existence of relics may lead to a tighter limit. In Ref. [3] a much weaker limit of $n<1.48$ from Hawking radiation constraints was found, which becomes tighter if relics are formed $(n<1.4$ if $T_{\mathrm{RH}}=10^{16} \mathrm{GeV}$ ). Our stronger constraint arises from our correcting of two errors in their paper, which both go the same way. The first is that we use the correct scaling law $\sigma(M) \propto M^{(1-n) / 4}$ for the variance at the horizon scale during radiation-domination, as mentioned earlier. The second is that our normalization to COBE is much higher. They omitted a numerical prefactor $\sqrt{512 \pi / 75}$ in the power spectrum expression, and also assumed that the normalization of $\delta_{\mathrm{H}}(k)$ (which from the four-year COBE data is $2 \times 10^{-5}$ ) and $\sigma(M)$ were interchangeable. In combination this raises the COBE normalization by a factor of over twenty. A much smaller additional correction is that the normalization from the COBE fouryear data 18 is higher than that from the first year's data 19].

\section{B. With a period of thermal inflation}

During thermal inflation the comoving Hubble radius $\left(H^{-1} / a\right)$ varies as $T$ so Eq. (12), for when a given comoving scale $k$ crosses the Hubble radius, becomes

$$
k^{-1}=\left(\frac{H^{-1}}{a}\right)=\left(\frac{H^{-1}}{a}\right)_{\mathrm{eq}}\left(\frac{T_{\mathrm{eq}}}{T}\right)\left(\frac{T_{\mathrm{ti}}}{10^{3} \mathrm{GeV}}\right),
$$

whilst the relation between horizon mass and temperature remains unchanged, since during inflation the horizon mass is constant so that for PBHs formed in between the two periods of inflation a given mass PBH will correspond to a larger scale than in the standard scenario. This leads to the modification of Eq. (14):

$$
\sigma_{\text {hor }}(M)=\sigma_{\text {hor }}\left(M_{\mathrm{eq}}\right)\left[\frac{M}{M_{\mathrm{eq}}}\left(\frac{10^{3} \mathrm{GeV}}{T_{\mathrm{ti}}}\right)^{2}\right]^{(1-n) / 4} .
$$

In this case the tightest constraint is $n<1.29$ from the deuterium destruction constraint evaluated at $M \sim$ $10^{10} \mathrm{~g}$, with all the constraints due to evaporation requiring $n<1.34$. In this case the constraints from gravitation and relics are weaker. The limit from the present-day density leads to $n<1.35$ for $M \sim 10^{15}$ g PBHs, which are diluted by the thermal inflation, and $n<1.49$ for $M \sim 10^{26} \mathrm{~g}$ PBHs, which are the lightest formed after thermal inflation. For $T_{\mathrm{RH}}<10^{14} \mathrm{GeV}$ the relics do not constrain $n$, since even if $\beta_{\mathrm{i}}$ is close to one the relics will be diluted away. However, for $T_{\mathrm{RH}}=10^{16} \mathrm{GeV}$ we find $n<1.3$ is required although this limit rapidly weakens as the reheat temperature falls towards $10^{14} \mathrm{GeV}$.

\section{The effect of non-gaussianity}

We now return to the issue of the gaussianity assumption used to obtain Eq. (7). Bullock and Primack [11] have stressed that the normal justification of gaussianity relies on the perturbations being very small, something which can no longer be justified when considering PBH formation. Unfortunately, the non-gaussian correction is strongly model dependent, and in detail must be examined case-by-case. They numerically study three different 'toy' models, in one case finding negligible nongaussianity but in the other two finding a very significant suppression in the number of large perturbations, which are of course exactly those utilized for PBH formation.

Expressed in terms of the probability of high density perturbations, the suppression can be very dramatic; in one of their toy models the 6-sigma perturbations are suppressed by a factor of $10^{150}$ ! However, despite that the effect on the constraint on $n$ is not large, because that asks a rather different question, namely how much larger does the variance $\sigma(M)$ have to be so that the 
non-gaussian perturbations reproduce the number density of gaussian ones? In their most extreme example, the answer is about three times; the perturbations with the appropriate number density correspond to about 3sigma perturbations in the non-gaussian case rather than the 9 -sigma or so perturbations of the gaussian case [11].

The conclusion then is that non-gaussian effects are model-dependent, and in the worst tested case weaken the constraints on $\sigma_{\text {hor }}(M)$ by about a factor three. Nongaussianity clearly cannot do much more than this, as the low required number density keeps us to the tail regardless of the amount of non-gaussianity. From Eq. (14), using the COBE normalization to keep $\sigma_{\text {hor }}\left(M_{0}\right)$ fixed, this weakens the constraint on $n$, in the worst case of non-gaussianity, by about 0.05 .

\section{CONCLUSIONS}

The interpretation of constraints on primordial black holes depends sensitively on the whole history of the universe from their time of formation. We re-examined the constraints assuming the standard radiation-dominated cosmology, and by correcting two errors in Carr et al. [3] found a significantly tighter constraint on the spectral index than they did, $n \lesssim 1.25$. This is in fact presently the tightest constraint on the spectral index, being somewhat stronger than large-scale structure constraints in the most general cosmologies, and much stronger than the constraint from distortions to the microwave background spectrum 20]. On the other hand, its application requires a belief that the spectral index remains constant over a much wider range of scales than the others, which is certainly possible but not mandatory. In general circumstances one must impose the general constraints on the formation rate we derived in Section IV.

We have analyzed the changes to the standard scenario brought about if a period of thermal inflation takes place in the early universe after the black holes form. Thermal inflation leads to a significant weakening on the constraint on the density perturbation spectrum. In Section IV we recomputed the constraints on the initial mass fraction of black holes; especially at low masses the constraints become very weak indeed. In Section V, we assumed a power-law spectrum of perturbations and constrained the spectral index $n$; we found the constraint weakened (relative to the standard cosmology) to $n \lesssim 1.3$. A novel additional feature is that thermal inflation predicts a missing mass range for black holes, extending up from $10^{18} \mathrm{~g}$ to $10^{26} \mathrm{~g}$. It will be hard to probe this range as black holes of these masses have negligible evaporation. However, if for some reason thermal inflation can start at a higher energy than currently supposed, say $10^{10} \mathrm{GeV}$, then the missing mass range could extend down into the evaporating regime.

It seems quite likely that density perturbations large enough to form PBHs will exhibit a significantly non- gaussian probability distribution, as emphasized by Bullock and Primack 11]. However, we have shown that this does not much alter the constraints; in the worst case it corresponds to a weakening of about 0.05 (regardless of whether or not thermal inflation occurred) which is comparable to the change due to thermal inflation. But typically the correction is expected to be quite a bit smaller than this [11], and hence smaller than the uncertainty in the cosmological model at early times.

\section{ACKNOWLEDGMENTS}

A.M.G. was supported by PPARC and A.R.L. by the Royal Society. A.R.L. thanks John Webb and UNSW for hospitality while some of this work was carried out. We thank Bernard Carr and Jim Lidsey for their comments, and Pedro Viana for useful discussions and providing the code for evaluation of $\sigma(M)$. We acknowledge use of the Starlink computer system at the University of Sussex.

[1] B. J. Carr, Astrophys. J. 205, 1 (1975); Ya. B. Zel'dovich, A. A. Starobinsky, M. Y. Khlopov and V. M. Chechetkin, Pis'ma Astron. Zh. 3, 308 (1977) [Sov Astron. Lett. 22, 110 (1977)]; S. Mujana and K. Sato, Prog. Theor. Phys. 59,1012 (1978); B. V. Vainer and P. D. Nasselskii, Astron. Zh 55, 231 (1978) [Sov. Astron. 22, 138 (1978)]; B. V. Vainer, D. V Dryzhakova and P. D. Nasselskii, Pis'ma Astron. Zh. 4, 344 (1978) [Sov. Astron. Lett 4,185 (1978)]; I. D. Novikov, A. G. Polnarev A. A. Starobinsky and Ya. B. Zel'dovich, Astron. Astrophys. 80, 104 (1979); D. Lindley, Mon. Not. R. Astron. Soc. 193, 593 (1980); T. Rothman and R. Matzner, Astrophys. Space. Sci 75, 229 (1981); J. H. MacGibbon, Nature 320, 308 (1987); J. H. MacGibbon and B. Carr, Astrophys. J. 371, 447 (1991).

[2] J. D. Barrow, E. J. Copeland and A. R. Liddle, Phys. Rev. D 46, 645 (1992).

[3] B. J. Carr, J. H. Gilbert and J. E. Lidsey, Phys. Rev. D 50, 4853 (1994).

[4] H. Kodama, M. Sasaki and K. Sato, Prog. Theor. Phys. 68, 1979 (1982); M. Crawford and D. N. Schramm, Nature 298, 538 (1982); S. D. H. Hsu, Phys. Lett. B 251, 343 (1990).

[5] B. J. Carr, Observational and Theoretical Aspects of Relativistic Astrophysics and Cosmology edited by J. L. Sanz and L. J. Goicoechea (World Scientific, Singapore, 1985).

[6] B. J. Carr and J. E. Lidsey, Phys. Rev. D48, 543 (1993).

[7] D. H. Lyth and E. D. Stewart, Phys. Rev. Lett. 75, 201 (1995).

[8] G. D. Coughlan, W. Fischler, E. W. Kolb, S. Raby and G. G. Ross, Phys. Lett. B131, 59 (1983); J. Ellis, D.V. Nanopoulos and M. Quiros, Phys. Lett. B174, 176 (1986); B. de Carlos, J. A. Casas, F. Quevedo and E. 
Roulet, Phys. Lett. B318, 447 (1993); T. Banks, D. B. Kaplan and A. E. Nelson, Phys. Rev. D49, 779 (1994); L. Randall and S. Thomas, Nucl. Phys. B449, 229 (1995); T. Banks, M. Berkooz and P. J. Steinhardt, Phys. Rev. D52, 705 (1995); M. Dine, L. Randall and S. Thomas, Phys. Rev. Lett. 75, 398 (1995).

[9] D. H. Lyth and E. D. Stewart, Phys. Rev. D 53, 1784 (1996).

[10] T. Barreiro, E. J. Copeland, D. H. Lyth and T. Prokopec, Phys. Rev. D 54, 1379 (1996).

[11] J. S. Bullock and J. R. Primack, to appear, Phys. Rev. $\mathrm{D}$, astro-ph/9611106.

[12] E. R. Harrison, Phys. Rev. D1, 2726 (1970).

[13] B. J. Carr, Astrophys. J. 201, 1 (1975).

[14] A. R. Liddle and D. H. Lyth, Phys. Rep. 231, 1 (1993).

[15] D. N. Page, Phys. Rev. D 13, 198 (1976).

[16] M. J. Bowick, S. B. Giddings, J. A. Harvey, G. T. Horowitz and A. Strominger, Phys. Rev. Lett. 61, 2823 (1988); S. Coleman, J. Preskill, and F. Wilczek, Mod. Phys. Lett. A 6, 1631 (1991); G. W. Gibbon and K. Maeda, Nucl. Phys. B298, 741 (1988); D. Garfinkle, G. T. Horowitz and S. Strominger, Phys. Rev. D 43, 3140 (1991); J. Preskill, P. Schwarz, A. Shapere, S. Trivedi and F. Wilczek Mod. Phys. Lett. A 6, 2353 (1991).

[17] E. F. Bunn, A. R. Liddle and M. White, Phys. Rev. D 54, 5917 (1996); see also E. F. Bunn and M. White, to appear, Astrophys. J, astro-ph/9607060.

[18] K. Gorski et al., Astrophys. J. 464, L11 (1996).

[19] U. Seljak and E. Bertschinger, Astrophys. J. 417, L9 (1993)

[20] W. Hu, D. Scott and J. Silk, Astrophys. J. 430, L5 (1994). 